\title{
Solvation of Fluorinated Single-Wall Carbon Nanotubes in Alcohol Solvents
}

\author{
E. T. Mickelson, ${ }^{\dagger}$ I. W. Chiang, ${ }^{\dagger}$ J. L. Zimmerman, ${ }^{\dagger}$ P. J. Boul, ${ }^{\dagger}, \sharp$ J. Lozano,${ }^{\S}$ J. Liu,,$\dagger$ \\ R. E. Smalley, ${ }^{\dagger}$, R. H. Hauge, ${ }^{\dagger}$ and J. L. Margrave ${ }^{* \dagger}$ \\ Rice Quantum Institute and Department of Chemistry, MS-60, Rice University, 6100 Main Street, \\ Houston, Texas 77005, Center for Nanoscale Science and Technology and Department of Physics, MS-100, \\ Rice University, 6100 Main Street, Houston, Texas 77005, and Center for Materials Chemistry, Department of \\ Chemistry and Biochemistry, University of Texas at Austin, Austin, Texas 78712
}

Received: November 30, 1998; In Final Form: March 5, 1999

\begin{abstract}
Highly purified single-wall carbon nanotubes (SWNTs) were fluorinated to form "fluorotubes", which were then solvated as individual tubes in various alcohol solvents via ultrasonication. The solvation of individual fluorotubes was verified by dispersing the tubes on a mica substrate and examining them with atomic force microscopy (AFM). Elemental analysis of the tubes reveals that light sonication in alcohol solvents does not remove significant amounts of the fluorine. While these solutions are metastable, they will persist long enough (over a week) to permit solution-phase chemistry to be carried out on the fluorotubes. For example, the solvated fluorotubes can be precipitated out of solution with hydrazine to yield normal, unfluorinated SWNTs, or they can be reacted with sodium methoxide to yield what are apparently methoxylated SWNTs. These reaction products have been examined with elemental analysis and a variety of spectroscopies and microscopies.
\end{abstract}

\section{Introduction}

Since the discovery of single-wall carbon nanotubes (SWNTs) in $1993,{ }^{1}$ researchers have been searching for ways to manipulate them chemically. While there have been many reports and review articles on the production and physical properties of carbon nanotubes, ${ }^{2-9}$ reports on chemical manipulation of nanotubes have been slow to emerge. There have been reports of functionalizing nanotube ends with carboxylic groups ${ }^{10,11}$ and then further manipulation to tether them to gold particles via thiol linkages. ${ }^{12}$ While theoretical results have suggested that functionalization of the nanotube side wall is possible, ${ }^{13}$ only recently has experimental evidence of this been presented.

We have reported on the side wall functionalization of SWNTs by reacting them with elemental fluorine. ${ }^{14}$ In that investigation we discovered that fluorine could be added to the side wall of carbon nanotubes, yielding stoichiometries of approximately $\mathrm{C}_{2} \mathrm{~F}$ without destruction of the tubelike structure. Haddon and co-workers have reported solvating SWNTs by adding octadecylamine groups on the ends of the tubes and then adding dichlorocarbenes to the nanotube side wall, albeit in relatively low quantities $(\sim 2 \%){ }^{15}$

We report here on the high degree of solvation that can be achieved by sonicating fluorinated SWNTs in a variety of alcohol solvents. We also show that reactions can be carried out on these nanotubes while in solution by reacting them with hydrazine, which serves as a defluorinating agent. This is an extension of the defluorinating procedure we have described previously. ${ }^{14}$ We further show that these "fluorotubes" can be reacted with sodium methoxide (a strong nucleophile) while in solution to form what are apparently methoxylated SWNTs.

\footnotetext{
* Corresponding author. E-mail: margrav@ rice.edu.

$\dagger$ Rice Quantum Institute and Department of Chemistry, Rice University.

$\doteqdot$ Center for Nanoscale Science and Technology and Department of Physics, Rice University.

$\S$ University of Texas at Austin.
}

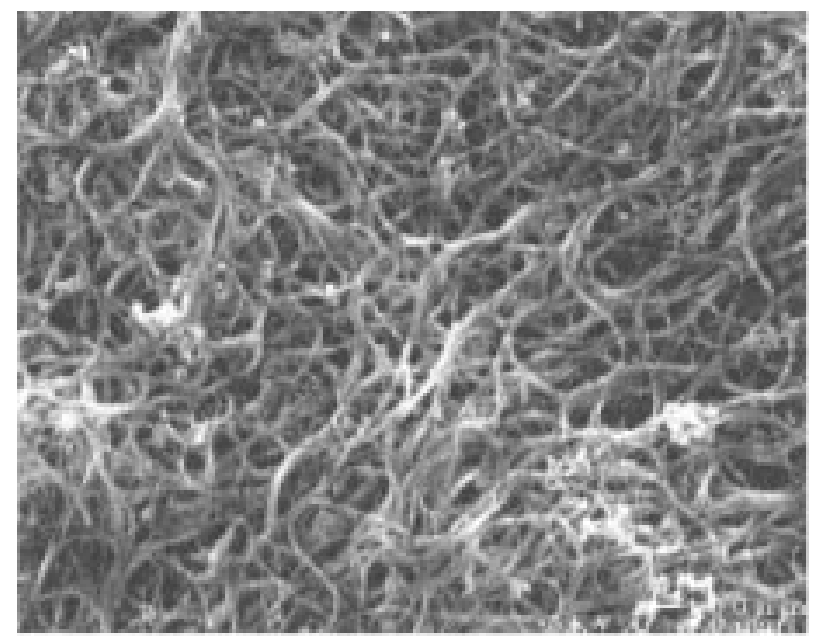

Figure 1. SEM image of purified SWNTs.

\section{Experimental Section}

2.1. Preparation of Highly Purified SWNTs. Single-wall carbon nanotubes were produced by the dual pulsed laser vaporization of $\mathrm{Co} / \mathrm{Ni}$ doped graphite rods and purified by methods discussed previously. ${ }^{16}$ The SWNTs produced in this way are primarily $(10,10)$ nanotubes. The purified product is a metastable colloidal suspension of SWNT "ropes"17 (bundles of tubes ranging from a few to 200 SWNTs) in a $0.2 \%$ aqueous solution of Triton X-100 surfactant. This was then filtered over a PTFE filter membrane (Sartorius, with $0.2 \mu \mathrm{m}$ pore dimensions) and rinsed with methanol. Filtering this and rinsing with methanol lead to a final product that is a free-standing "mat" or "bucky paper" of SWNTs that is approximately $10 \mu \mathrm{m}$ thick. Purity of the SWNTs was monitored via scanning electron microscopy (JEOL 6320F SEM). Figure 1 shows a sample of typical purity. This product was then resuspended by sonication in dimethylformamide (DMF; Fisher, HPLC grade). Such 
treatment is believed to "cut" the tubes at their defect sites ${ }^{18}$ and also seems to unravel the ropes somewhat, leading to bundles containing fewer SWNTs. This product was then filtered, rinsed, and heated in an oven at $150{ }^{\circ} \mathrm{C}$ for $2 \mathrm{~h}$ prior to fluorination. We believe that this last step (sonication in DMF) results in smaller SWNT ropes and ultimately leads to a more efficient fluorination.

2.2. Preparation of Fluorinated SWNTs. The purified nanotubes $(5-10 \mathrm{mg}$ in the form of bucky paper) were then placed in a temperature-controlled fluorination reactor constructed of Monel and nickel. After sufficient purging in $\mathrm{He}$ (Trigas $99.995 \%$ ) at $250{ }^{\circ} \mathrm{C}$, fluorine (Air Products $98 \%$, purified of $\mathrm{HF}$ by passing it over NaF pellets) was introduced. The fluorine flow was gradually increased to a flow rate of $2 \mathrm{sccm}$ diluted in a He flow of $20 \mathrm{sccm}$. The fluorination was allowed to proceed for approximately $10 \mathrm{~h}$ at which point the reactor was brought to room temperature and the fluorine flow was gradually lowered. After the fluorine flow was completely halted, the reactor was purged at room temperature for approximately $30 \mathrm{~min}$ before removing the fluorinated product. The fluorinated SWNTs consisted of approximately 70 atom $\%$ carbon and 30 atom \% fluorine as determined by electron microprobe analysis (EMPA; Cameca SX-50). This fluorinated product has been well characterized with Raman, IR, SEM, TEM, resistance measurements, and, for this work, X-ray photoelectron spectroscopy (Physical Electronics PHI 5700 XPS

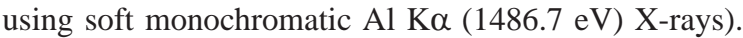

2.3. Solvation in Alcohols. Sonication of the fluorinated SWNT material in alcohols was carried out by placing approximately $1 \mathrm{mg}$ of material into a vial containing approximately $10 \mathrm{~mL}$ of alcohol solvent and sonicating for approximately $10 \mathrm{~min}$. Sonication was performed by partially immersing the capped vial in a Cole-Parmer ultrasonic cleaner (containing water) operating at $55 \mathrm{kHz}$. The solvated fluorotubes were then dispersed on a clean mica surface by means of a 3000 rpm rotary spinner (Headway Research, Inc.) and examined with atomic force microscopy (Digital Instruments Multimode SPM). The solvated fluorotubes were also analyzed with a Shimadzu model 1601PC UV-vis spectrometer using quartz cuvettes.

The solvated fluorotubes were filtered over a $0.2 \mu \mathrm{m}$ PTFE filter. Once dry, the fluorotubes could be peeled off the paper to form a free-standing film. This film was then examined with Raman spectroscopy (Jobin Yvon-Spex model HR 460 monochromator coupled with an ISA Spectrum ONE series CCD detector and using $514.5 \mathrm{~nm}$ excitation from a Liconix Ar laser) and with EMPA to determine whether any reaction had taken place on the basis of the composition of the product.

2.4. Reactions in Solution. Anhydrous hydrazine (Aldrich, 98\%) was added to the solvated fluorotubes. The reaction mixture was continually stirred with a glass stir bar for a period of about an hour. The reaction mixture was filtered, rinsed with methanol, and allowed to dry. This product was then examined with EMPA and Raman spectroscopy. It was also suspended in dimethylformamide, dispersed on a mica surface, and examined with AFM.

Fluorotubes were also sonicated in a $0.5 \mathrm{M}$ sodium methoxide in methanol solution (Aldrich, ACS reagent) for approximately $10 \mathrm{~min}$. The tubes broke up and appeared to be suspended but quickly fell out of solution upon standing. This too was filtered, rinsed, and examined with EMPA and EI mass spectroscopy (Finnigan MAT 95).

\section{Results and Discussion}

3.1. Solvation in Alcohols. Our efforts to solubilize fluorotubes began with the "like dissolves like" approach of sonicating

\section{SCHEME 1}

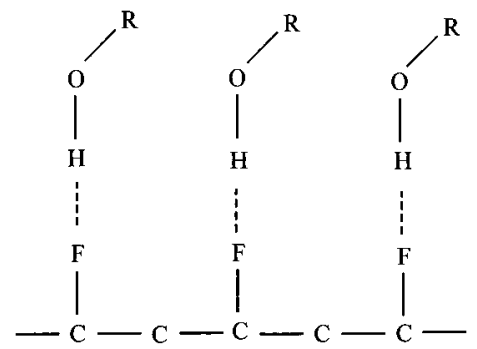

and heating them in perfluorinated solvents. This met with little success, however. We then turned our attention to the possibility of solvating them in hydrogen-bonding solvents. Recent studies of the hydrogen-bonding capabilities of alkyl fluorides suggest that the fluorine in such species are poor hydrogen bond acceptors. ${ }^{19,20}$ The $\mathrm{F}^{-}$ion, however, is one of the best hydrogen bond acceptors available. The strength of the hydrogen bond formed between $\mathrm{HF}$ and $\mathrm{F}^{-}$approximates that of a covalent bond. ${ }^{21}$ An XPS analysis of our fluorinated SWNT product reveals an F 1s peak at a binding energy of $687 \mathrm{eV}$. Poly(tetrafluoroethylene) has an F 1s binding energy of $691.5 \mathrm{eV}$. This suggests that the fluorine bonded to the fluorotubes is considerably more ionic than the fluorine present in alkyl fluorides. ${ }^{22}$ Thus, the increased ionic nature of the $\mathrm{C}-\mathrm{F}$ bond in the fluorotubes may make the fluorine on it better hydrogen bond acceptors.

Sonicating the fluorotubes in alcohol solvents produced metastable solutions. These solutions were stable for a couple of days to over 1 week, depending on the concentration and solvent used. Alcohol solvents used included methanol, ethanol, 2,2,2-trifluoroethanol, 2-propanol, 2-butanol, $n$-pentanol, $n$ hexanol, cyclohexanol, and $n$-heptanol. While typical sonication times were around $10 \mathrm{~min}$, the heavier solvents (pentanol and up) required slightly longer sonication times in order to fully suspend the tubes. Of the solvents used, 2-propanol and 2-butanol seemed to solvate the fluorotubes the best with the solutions being stable for more than a week. The solubility limit of the solvated "fluorotubes" in 2-propanol is at least $1 \mathrm{mg} /$ $\mathrm{mL}$. This solution was stable for slightly less than a week with some particulate matter precipitating out after a few days. This suggests that pushing the solubility limit somewhat decreases the solution's stability or that a supersaturated solution can exist for a shorter period of time. All of the other solutions were stable for at least a couple of days before the onset of precipitation. A likely scenario for such solvation would be hydrogen bonding between the alcohol's hydroxyl hydrogen and the nanotube-bound fluorine (Scheme 1). No evidence of alkoxy substitution (or evolution of HF) was observed.

We also made efforts to solvate the fluorotubes in other strong hydrogen-bonding solvents such as water, diethylamine, acetic acid, and chloroform. While water will not "wet" the fluorotube by itself, it will with the addition of a small amount of acetone. Still, even long sonication times in this water/acetone mixture failed to solvate the fluorotubes. Likewise, neither diethylamine nor acetic acid would solvate the fluorotubes. Chloroform solvated the tubes, but the solution was far less stable than those in alcohol solvents with the fluorotubes falling out of solution in less than an hour.

Fluorotubes from all of the solutions (except those in cyclohexanol, $n$-hexanol, and $n$-heptanol) were examined with atomic force microscopy. Figure 2 shows an AFM scan of fluorotubes that had been dissolved in 2-butanol and then dipersed on a clean mica surface. This result is fairly typical of 


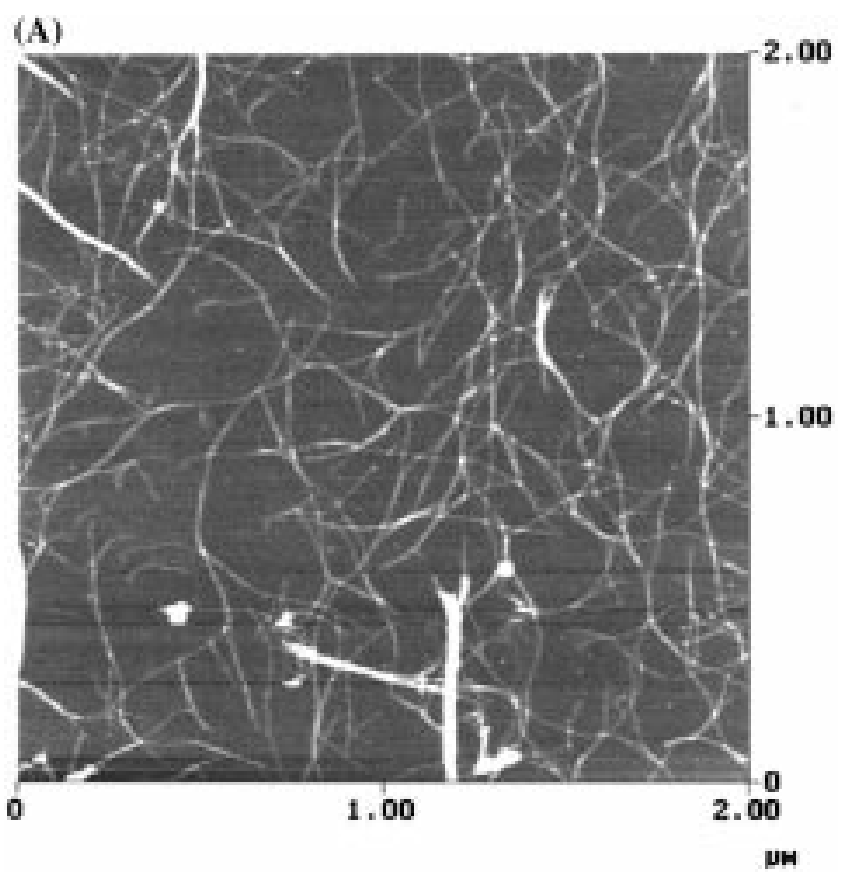

(B)

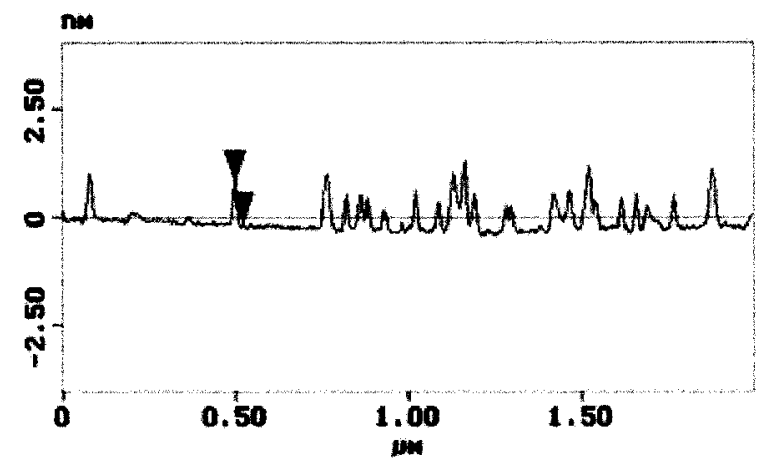

Figure 2. (A) AFM image of fluorotubes that have been dissolved in 2-butanol and dispersed on mica. (B) Typical height analysis of the scan in (A), revealing the tube diameters to be on the order of 1.2-1.4 $\mathrm{nm}$, values on the order of those determined previously for this product using TEM and XRD.

all the fluorotube/alcohol solutions that we examined with AFM. Almost all the tubes are believed to be solvated, as few "ropes" (bundles of tubes) are present.

We examined some of these solutions with ${ }^{19} \mathrm{~F}$ NMR, but this proved to be rather uninformitive. It yielded a broad peak centered at around $-175 \mathrm{ppm}$. While this is indicative of fluorine being present, the broadening is due to either a wide variety of $\mathrm{F}$ environments (as seen in the inhomogeneous fluorination of $\left.\mathrm{C}_{60}\right)^{23}$ or insufficient "tumbling" while in solution. No information regarding the possible hydrogenbonding environments could be obtained with this method.

Filtering a solution of fluorotubes in isopropyl alcohol over a PTFE filter and examining the tubes with EMPA revealed no presence of oxygen and only slightly lower fluorine levels $(\mathrm{C} / \mathrm{F}$ atomic percent ratio $=72 / 28$ compared with $70 / 30$ for the starting material). This would suggest that the solvation process is not the result of a chemical reaction but is instead the result of hydrogen bonding between the alcohol and the fluorines on the nanotube surface. Analysis of fluorotubes sonicated for much longer times $(2 \mathrm{~h})$ showed reduced levels of fluorine $(\mathrm{C} / \mathrm{F}$ atomic percent ratio $=76 / 24$ ), yet they remained solvated. Apparently, ultrasonication can lead to removal of some of the fluorine if allowed to progress long enough. The fluorotubes were sonicated

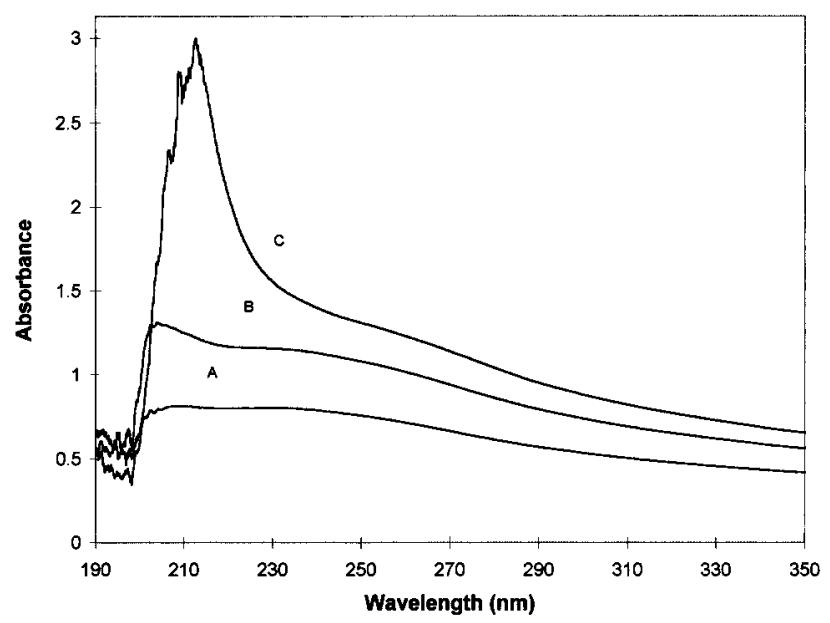

Figure 3. UV spectrum of fluorotubes solvated in 2-propanol after sonication times of (A) $10 \mathrm{~min}$, (B) $40 \mathrm{~min}$, and (C) $130 \mathrm{~min}$.

continuously in 2-propanol and monitored with UV-vis absorption spectroscopy for sonication time $t=10 \mathrm{~min}$ and every 30 min after that. After sonication for 40 min the solution exhibited an absorption band at $204 \mathrm{~nm}$. This band continued grow and to red-shift to lower energy as the sonication proceeded and fluorine was presumably being eliminated. After sonication for $130 \mathrm{~min}$, the peak had increased in intensity and shifted to 237 nm (Figure 3).

3.2. Reactions in Solution. Previous studies have shown that hydrazine acts as an effective defluorinating agent. ${ }^{14}$ Adding anhydrous hydrazine to a solution of fluorotubes in 2-propanol caused them to immediately precipitate out of solution. Filtering the solution after letting it sit for an hour yielded a product of very low fluorine content, as determined by EMPA $(\mathrm{C} / \mathrm{F}$ atomic percent ratio $=93 / 7)$. Unreacted SWNTs are known to suspend fairly well in DMF. ${ }^{18}$ Suspending this product in DMF and dispersing it on a mica surface followed by AFM analysis yielded tubes very reminiscent of the starting material (Figure 4).

Since the Raman spectroscopy of SWNTs has been well established, ${ }^{24-26}$ we utilized it as a probe to follow the starting material through the fluorination, sonication, and defluorination. Raman spectroscopy on the hydrazine-defluorinated product yields a spectrum similar to that of the starting material and very different from those of the fluorinated SWNTs (Figure 5).

Sonication of the fluorotubes in a sodium methoxide in methanol solution for $2 \mathrm{~h}$ resulted in the tubes precipitating out of solution. After the filtered product was rinsed with water (to remove $\mathrm{NaF}$ ) and methanol, then dried in an oven at $140{ }^{\circ} \mathrm{C}$ for half an hour, it was analyzed with EMPA, which revealed the $\mathrm{C} / \mathrm{F} / \mathrm{O}$ relative atomic percent to be $79 / 17 / 4$. This varies considerably from the starting material, which had $\mathrm{C} / \mathrm{F} / \mathrm{O}$ relative atomic percents of 66/33.7/0.3, and suggests a product soichiometry of $\mathrm{C}_{4.4} \mathrm{~F}\left(\mathrm{OCH}_{3}\right)_{0.25}$. Pyrolysis of this product with a hightemperature probe inside a mass spectrometer followed by electron ionization yielded significant quantities of methoxy ions $(\mathrm{m} / \mathrm{z}=31)$ coming off primarily at $650-700^{\circ} \mathrm{C}$ as determined by the residual ion current trace. The high temperature for evolution indicates that the methoxy groups seen were originally strongly bonded to the nanotube. If the oxygen ratios seen in the EMPA are reflective of the number of methoxy groups present on the nanotube, we would conclude that the majority of these would have to be bonded to the nanotube side wall on the basis of the fact that the number of nanotube end carbons is extremely small relative to the number of side wall carbons. 


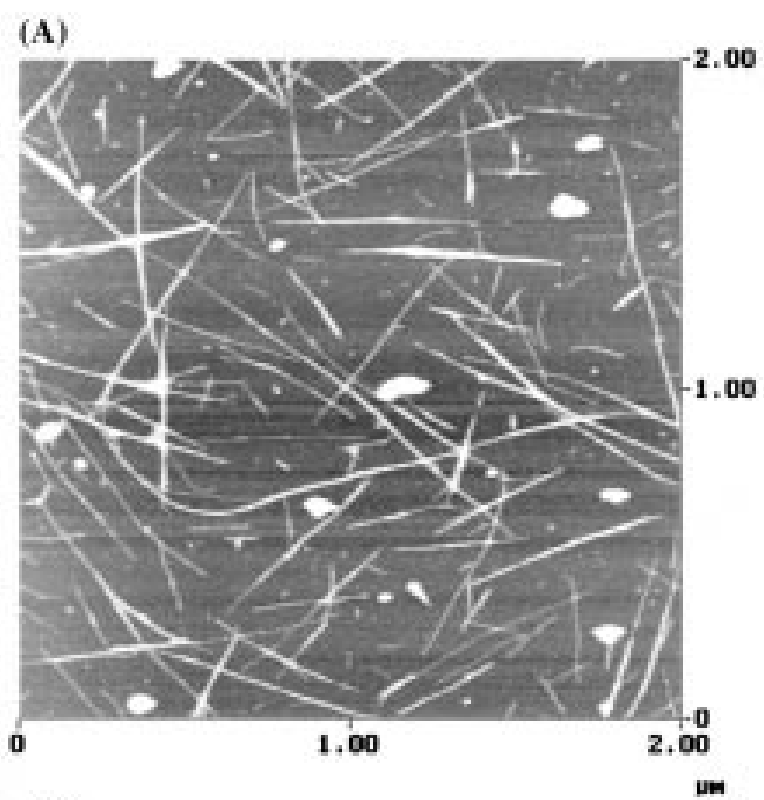

(B)

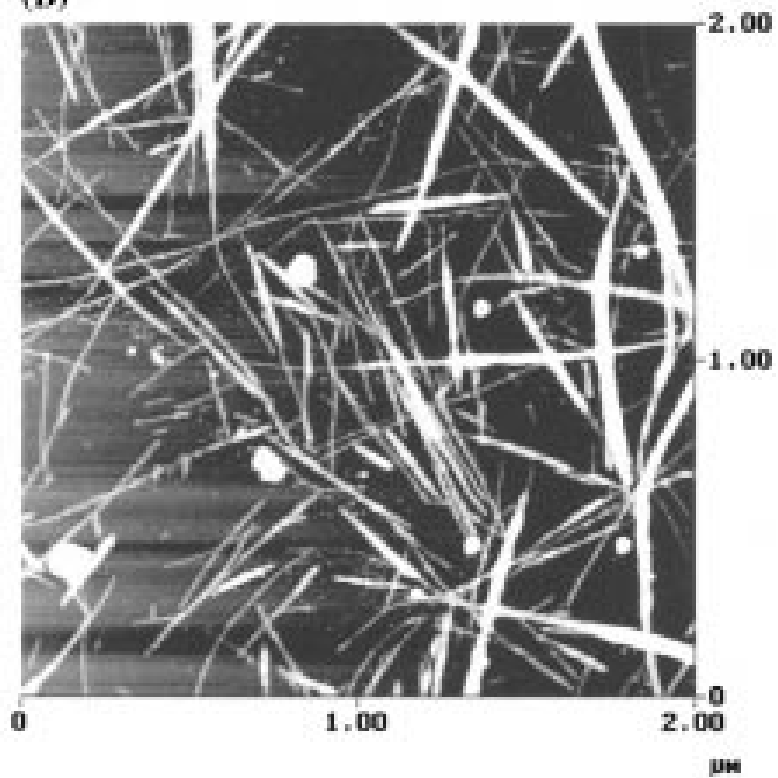

Figure 4. (A) AFM image of fluorotubes after having been defluorinated with $\mathrm{N}_{2} \mathrm{H}_{4}$, filtered, resuspended in DMF, and dispersed on mica. (B) AFM image of untreated SWNTs dispersed on mica.

Nucleophilic attack on the fluorinated nanotube by a methoxy anion is a plausible scenario, since nucleophilic attack of this type has been well documented in the case of fluorinated fullerenes. ${ }^{27,28}$ The $\mathrm{C}-\mathrm{F}$ bonds on fluorinated fullerenes (and carbon nanotubes) are weakened relative to the $\mathrm{C}-\mathrm{F}$ bonds in alkyl fluorides owing to an "eclipsing strain effect". ${ }^{29}$ A nucleophilic attack of this type is likely to occur via attack on an electropositive carbon beta to a carbon with a fluorine attached to it as shown in Scheme 2. This is rationalized by the fact that an $\mathrm{S}_{\mathrm{N}} 1$ type substitution is energetically unfavorable and that backside attack, as in an $\mathrm{S}_{\mathrm{N}} 2$ type mechanism, is impossible. ${ }^{30}$

\section{Conclusions}

We have shown that single-wall carbon nanotubes can be fluorinated and then sonicated in alcohols to form stable solutions of fluorotubes. This solvation allows one to manipulate the fluorotubes in ways that were previously unavailable and
(A)

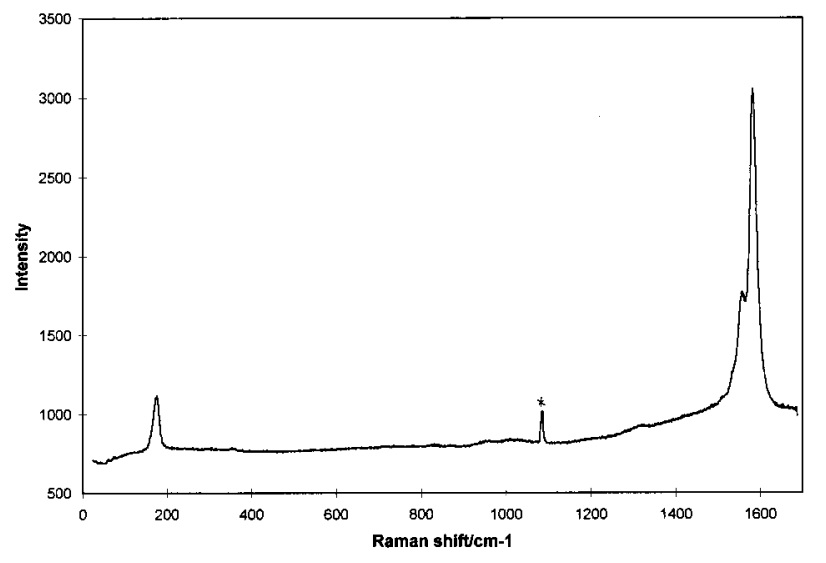

(B)

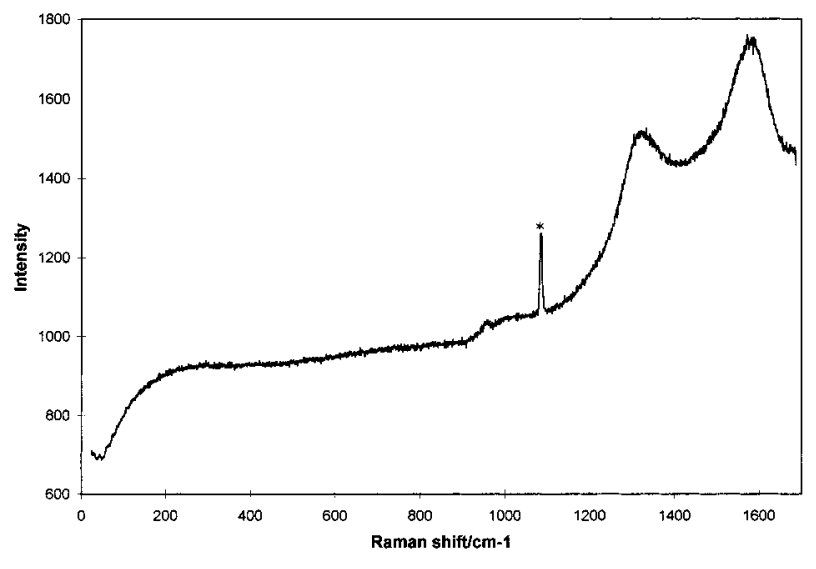

(C)

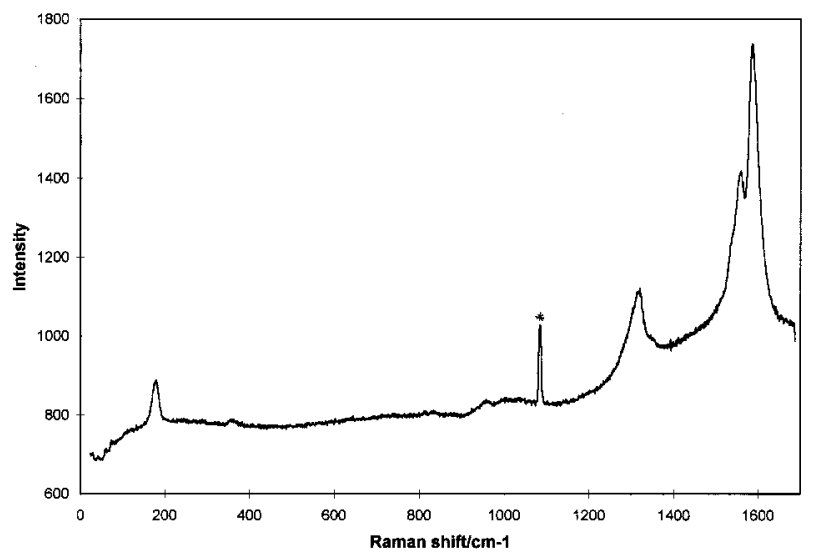

Figure 5. (A) Raman spectrum of pure, untreated SWNTs. (B) Raman spectrum of fluorotubes. (C) Raman spectrum of fluorotubes after having been defluorinated with $\mathrm{N}_{2} \mathrm{H}_{4}$. * denotes Ar plasma line.

\section{SCHEME 2}

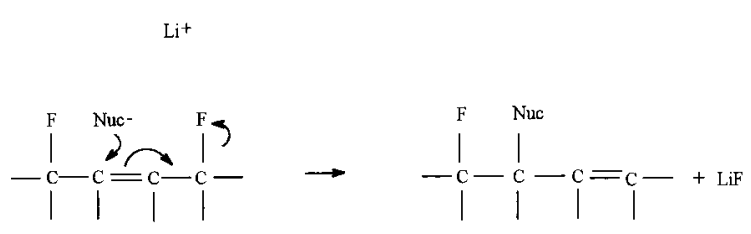

opens the door to a wide variety of possibilities with respect to exploration of the physical and chemical properties of fluorotubes. "Tuning" the fluorine content of a fluorotube by first fluorinating it heterogeneously, solvating it in an alcohol, and then defluorinating it with substoichiometric quantities of 
hydrazine becomes a reasonable way of making a wide variety of fluorotubes with differing fluorine contents and, presumably, quite different properties.

We have further demonstrated that once solvated, these fluorotubes can then be reacted with species while in solution to either defluorinate or further functionalize them. While we have only begun to explore the chemistry possible with these solvated fluorotubes, we believe that this could become an important route to the synthesis of a wide variety of functionalized nanotubes having many different and useful properties.

Acknowledgment. We thank Milton Pierson for his assistance with the EMPA, Terry Marriot for help with the mass spectroscopy, Larry Alemany for his assistance in getting the NMR data, and Jane Bertone and Professor Vicki Colvin for their assistance in obtaining the Raman spectra. This work was supported by grants from the Robert A. Welch Foundation and the ONR (BMDO). J. Lozano acknowledges instrumentation support provided through the NSF-supported Science and Technology Center of the University of Texas at Austin (Grant CHE 8920120).

\section{References and Notes}

(1) Iijima, S.; Ichihashi, T. Nature 1993, 363, 603-605.

(2) Ebbesen, T. W. J. Phys. Chem. Solids 1997, 58 (11), 1979-1982.

(3) Yakobson, B. I.; Smalley, R. E. Am. Sci. 1997, 85, 324-337. 15038

(4) Charlier, J.-C.; Lambin, Ph. Phys. Rev. B 1998, 57 (24), 15037-

(5) Cowley, J. M.; Nikolaev, P.; Thess, A.; Smalley, R. E. Chem. Phys. Lett. 1997, 265, 379-384.

(6) Tohji, K.; Takahashi, H.; Shinoda, Y.; Shimizu, N.; Jeyadevan, B.; Matsuoka, I.; Saito, Y.; Kasuya, A.; Ito, S.; Nishina, Y. J. Phys. Chem. B 1997, 101, 1974-1978.

(7) Bandow, S.; Rao, A. M.; Williams, K. A.; Thess, A.; Smalley, R. E.; Eklund, P. C. J. Phys. Chem. B 1997, 101, 8839-8842.

(8) Tans, S. J.; Devoret, M. H.; Dai, H.; Thess, A.; Smalley, R. E.; Geerligs, L. J.; Dekker, C. Nature 1997, 386, 474-477.

(9) Bandow, S.; Asaka, S.; Saito, Y.; Rao, A. M.; Grigorian, L.; Richter,

E.; Eklund, P. C. Phys. Rev. Lett. 1998, 80 (17), 3779-3782

(10) Rao, C. N. R.; Govindaraj, A.; Satishkumar, B. C. Chem. Commun. 1996, $1525-1526$.
(11) Wong, S. S.; Joselevich, E.; Woolley, A. T.; Cheung, C. L.; Lieber, C. M. Nature 1998, 394, 52-55.

(12) Liu, J.; Rinzler, A. G.; Dai, H.; Hafner, J.; Bradley, R. K.; Boul, P.; Lu, A.; Iverson, T.; Shelimov, K.; Huffman, C. B.; Rodriguez-Macias, F.; Shon, Y.-S.; Lee, T. R.; Colbert, D. T.; Smalley, R. E. Science 1998, 280, 1253-1256.

(13) Cahill, P. A.; Rohlfing, C. M. Tetrahedron 1996, 52 (14), 52475256.

(14) Mickelson, E. T.; Huffman, C. B.; Rinzler, A. G.; Smalley, R. E.; Hauge, R. H.; Margrave, J. L. Chem. Phys. Lett. 1998, 296 (1-2), 188194.

(15) Chen, J.; Hamon, M. A.; Hu, H.; Chen, Y.; Rao, A. M.; Eklund, P. C.; Haddon, R. C. Science 1998, 282, 95-98.

(16) Rinzler, A. G.; Liu, J.; Dai, H.; Nikolaev, P.; Huffman, C. B Rodriguez-Macias, F. J.; Boul, P. J.; Lu, A. H.; Heymann, D.; Colbert, D. T.; Lee, R. S.; Fischer, J. E.; Rao, A. M.; Eklund, P. C.; Smalley, R. E. Appl. Phys. A 1998, 67, 9-37.

(17) Thess, A.; Lee, R.; Nikolaev, P.; Dai, H.; Petit, P.; Robert, J.; Xu, C.; Lee, Y.-H.; Kim, S.-G.; Rinzler, A. G.; Colbert, D. T.; Scuseria, G. E.; Tomanek, D.; Fischer, J. E.; Smalley, R. E. Science 1996, 273, 483-487.

(18) Liu, J.; Smalley, R. E. Unpublished data.

(19) Dunitz, J. D.; Taylor, R. Eur. J. Chem. 1997, 3 (1), 89-98.

(20) Howard, J. A. K.; Hoy, V. J.; O’Hagan, D.; Smith, G. T. Tetrahedron 1996, 52 (38), 12613-12622.

(21) Harrell, S. A.; McDaniel, D. H. J. Am. Chem. Soc. 1964, 86, 4497.

(22) Watanabe, N.; Nakajima, T.; Touhara, H. Graphite Fluorides; Elsevier: Amsterdam, 1988; p 246.

(23) Kniaz, K.; Fischer, J. E.; Selig, H.; Vaughan, G. B. M.; Romanow, W. J.; Cox, D. M.; Chowdhury, S. K.; McCauley, J. P.; Strongin, R. M.; Smith, A. B. J. Am. Chem. Soc. 1993, 115, 6060-6064.

(24) Richter, E.; Subbaswamy, K. R. Phys. Rev. Lett. 1997, 79 (14), $2738-2741$.

(25) Rao, A. M.; Richter, E.; Bandow, S.; Chase, B.; Eklund, P. C.; Williams, K. A.; Fang, S.; Subbaswamy, K. R.; Menon, M.; Thess, A.; Smalley, R. E.; Dresselhaus, G.; Dresselhaus, M. S. Science 1997, 275, 187-191.

(26) Fang, S. L.; Rao, A. M.; Eklund, P. C.; Nikolaev, P.; Rinzler, A. G.; Smalley, R. E. J. Mater. Res. 1998, 13, 2405-2411.

(27) Mickelson, E. T.; Hauge, R. H.; Margrave, J. L. J. Fluorine Chem. 1998, 92 (1), 59-62.

(28) Taylor, R.; Holloway, J. H.; Hope, E. G.; Avent, A. G.; Langley, G. J.; Dennis, T. J.; Hare, J. P.; Kroto, H. W.; Walton, D. R. M. J. Chem. Soc., Chem. Commun. 1992, 665-667.

(29) Taylor, R. Russ. Chem. Bull., Engl. Ed. 1998, 47 (5), 823-832.

(30) Taylor, R. The Chemistry of the Fullerenes; Taylor, R., Ed.; World Scientific Publishing: London, 1995; pp 208-209. 